\title{
ACOUSTIC FEEDBACK CANCELLATION IN EFFICIENT HEARING AIDS USING GENETIC ALGORITHM
}

\author{
G. JAYANTHI*AND LATHA PARTHIBAN ${ }^{\dagger}$
}

\begin{abstract}
Many people are distracted from the normal life style, because of the hearing loss they have. Most of them do not use the hearing aids due to various discomforts in wearing it. The main and the foremost problem available in it is; the device introduces unpleasant whistling sounds, caused by the changing environmental noise, which is faced by the user daily. This paper describes the development of an algorithm, which focuses on the adaptive feedback cancellation, that improves the listening effort of the user. The genetic algorithm is one of the computational technique, that is used in enhancing the above features. The performance can also be compared with other comprehensive analysis methods, to evaluate its standards.
\end{abstract}

Key words: Hearing aids, Acoustic feedback, Feedback cancellation, Genetic algorithm

AMS subject classifications. $68 \mathrm{~W} 25$

1. Introduction. People with hearing loss, struggle to lead a normal healthy life, is considered to be $13 \%$ of world's population. Among them, hardly $20 \%$ will ever purchase a hearing aid. While of $32 \%$, may not wear hearing aids, though it is purchased. This is due to irritation and unpleasant whistling, caused by the changing enviromental noise, when the user enters into more noisy background. He is forced to hear a sudden and unpleasant sound under which the hearing becomes more complicated and restricts them from moving into a noisy background.

This irregular use of hearing aids leads to a variety of problems. The hearing aid system used for the hearing impaired people should be more natural, humanized and personalized as they likely to prefer the most suitable scheme for their own hearing compensation strategy [2].

Based on the degree of hearing threshold (Mild, Moderate and Severe), the type of loss (Conductive or Sensori-neural) is determined. The hearing thresholds are measured at each octave as $250 / 500 / 1 \mathrm{~K} / 2 \mathrm{~K} / 4 \mathrm{~K} / 8 \mathrm{~K}$ in a standard audiogram and the hearing loss is evaluated using pure tone audiometry [3]. Hence the hearing loss is characterized by the high thresholds in an audiogram is found out using the electro-acoustic charateristics, shown in Figure 1.1. To match the audiogram at high frequencies, large number of bands are to be used, which can be formed as the membership functions by the trial and error process, which is dealt later.

Hearing aid is an assist device, can be fitted in anyone or both the ears, which aids in hearing. The inner structure of the hearing aid is shown in Figure 1.2b. The hearing aid style is chosen with a particular degree of hearing loss, that is been fitted to users . Among them, the binuaral hearing aids offer more flexibility, contains all basic functions and much affordable. While the open-fit hearing aids have modified ear tips. The Receiver-in-canal is one of the open-fit hearing aids, in which the receiver is separated from the housing and kept at the ear tip (Figure 1.2.a).

2. Occlusion Effect. The occlusion effect is of occluding earmold or shell which leads to the unnatural, hollow sensation when the user is talking. Similarly annoying sensation of amplification called ampclusion. The utilization of vents of appreciable size is the most common and popular recommendation for hearing aids, dealing with the occlusion effect. Large vents are used to shunt a portion of low-frequency signals to the environment, enabling to remove all or part of the disturbing low-frequency own-voice elements. Whenever

\footnotetext{
* Sathyabama University, Department of Electronics and Communication Engineering, Chennai-600119, India (gujayanthi@ gmail.com ).

${ }^{\dagger}$ Pondicherry University Community College, Department of Computer Science Engineering, Pondicherry-605008, India (lathaparthiban@yahoo.com)
} 


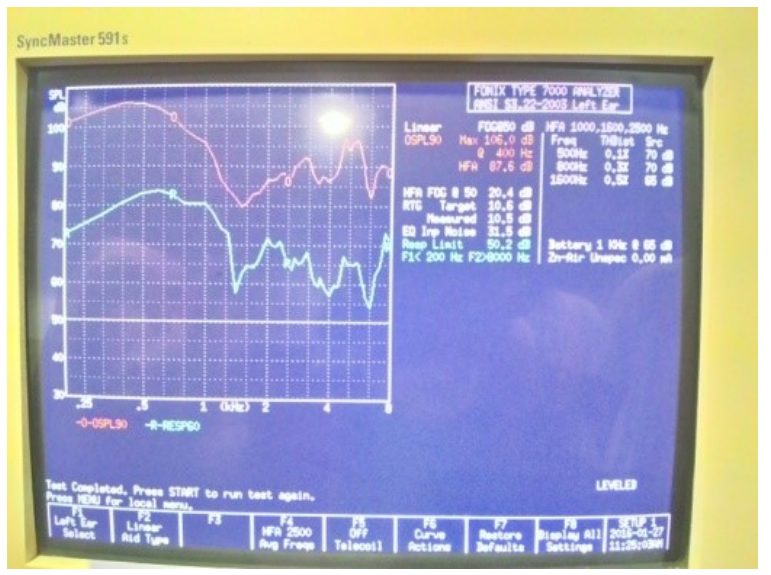

FIG. 1.1. Electro Acoustic Characteristics

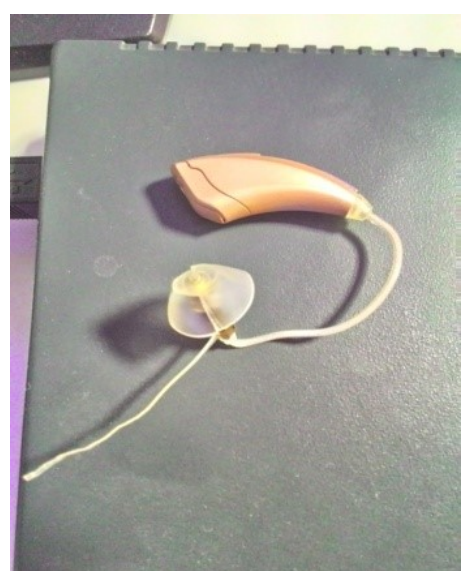

(a)

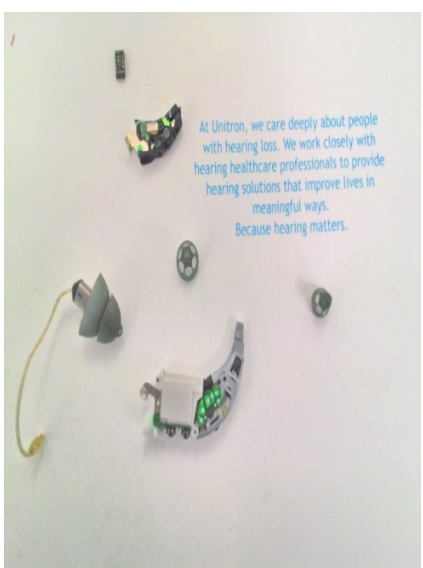

(b)

FIG. 1.2. (a) Receiver-In-Canal (b) Inner Structure of Hearing aid

occlusion effect problems occur, the vent shortening technique is recommended for use [8]. The occlusion effect is the perception of unnaturalness of the user's own voice, which makes them feel like talking in the barrel.

3. Acoustic Feedback. Feedback is a most common problem, which occurs in hearing aids as whistling or howling sounds. Normally the sound traverses from the microphone to the receiver, crossing the processing circuit Figure 3.1. When this processed sound after reaching the receiver, reverts back to the the microphone, primarilily through air, which creates the feedback path. This path leads to the hearing of unnecessary sounds and makes the hearer uncomfortable. These may occur sometimes through the bone and the soft tissues that

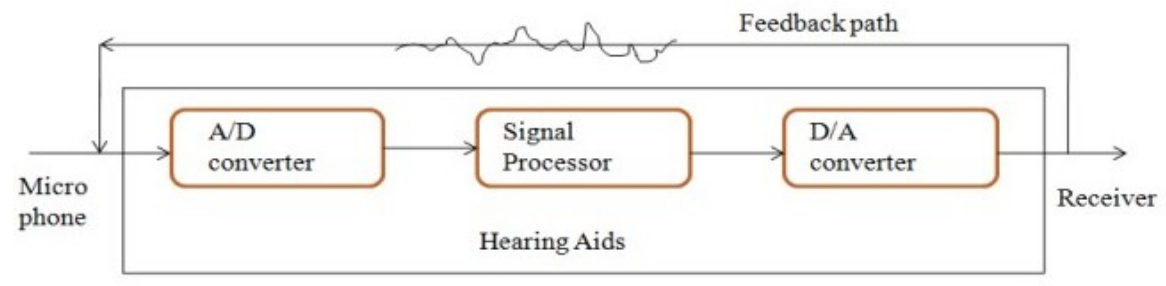

Fig. 3.1. Feedback Path Tansmission 


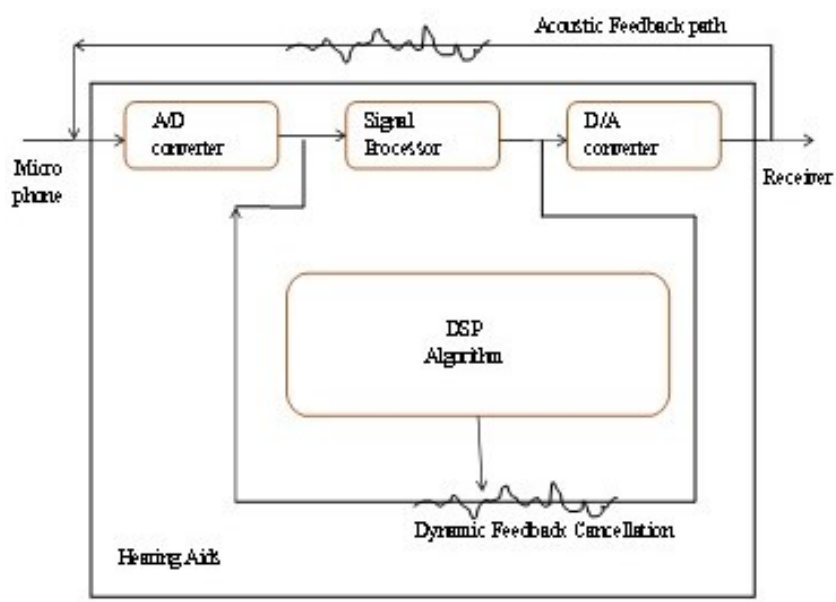

FIG. 3.2. Dynamic Feedback Cancellation

surrounds the hearing aid [5].

The feedback is mainily initiated during chewing, yawning or bringing any electronic gadgets nearer to ear device. While occlusion effect is the trapping of sounds in the ear canal, when it is occluded. The feedback and the occlusion effect both imposes a great controversy as the feedback can be reduced by decreasing the vent size while the occlusion effect may be eliminated by increasing the size of the vent [12].

Feedback effect that occurs in ear is known for Acoustic Feedback, which occurs when the high frequency tonal sounds emitted by hearing aids. It happens only when the amplification factor of the forward path signals are much greater than the attenuation factor in the feedback path, by which the hearing aid is lead into oscillation. When the amplification factor is set just closer or below the attenuation factor, a sub-ocillatory feedback is generated. This includes the peaks and valleys in the frequency response of the hearing aid, which degrades the speech quality and intelligibilty.

The feedback effect is cancelled in many hearing aids, by reducing the frequency-specific gain when the output level exceeds the frequency-specific threshold. Also this happens when the closed loop gain exceeds unity for the corresponding frequency, at that particular time. A popular LMS filtering is used to cancel the signal components with specific correlation properties.

The main sources of feedback can be due to talking, chewing or yawning, etc. In multichannel hearing aids, a wide range dynamic compression method is used. Figure 3.2. This is done by providing more gain for lower level sounds and minimum gain for higher level sounds. But still the feedback limits the amount of gain that can be provided by a hearing device. Additionally, the presence of feedback in a hearing aid limits the style of wearing, which makes the user to avoid wearing the device, particularly those who want to conceal their hearing loss.

The general methods to reduce acoustic feedback are: (i) Reducing the gain of forward path at one or more high frequecy bands in which feedback occurs (ii) Increasing the attenuation level in the feedback path. This is done by decreasing the vent size to an extent.

4. Literature Survey. Soon-Suck Jarng has divided the noise reduction algorithm into two parts. One is to estimate the environmental noise and the other is to enhance the amplifier to improve voice audibility over the estimated noise. A noise spectrum is calculated from the measured signal during the absence of speech activity, using spectral subtraction method. In this paper, it is found that whenever the noise spectrum is estimated to be too steep, some musical noise is generated and if it is estimated to be too low, the input noise speech is distorted [16].

A.H.Kparsi proposed a novel noise power spectral density estimator for binaural hearing aids operating in a diffuse noise field environment which can work for colored noise in a diffuse noise field. Although it does not require a voice activity detection, the noise power spectrum can be estimated irrespective of the speech 
activity. No noise tracking latency is experienced and the direction of arrival of the source speech signal can be arbitrary. The proposed noise estimator can be combined with any hearing aid noise reduction technique, where the accuracy of the noise estimation can be critical to achieve a satisfactory de-noising performance. However the method requires a binuaral system, which requires access to left and right noisy signals [17].

Y.F.Chiang has introduced a novel pitch based formant estimation algorithm and an architecture for the adaptive feedback cancellation for the hearing aid applications. The system consists of a peak and harmonic detection in the forward path, includes the decorrelation filter coefficients update and an adaptive feedback cancellation in backward path with a pitch based detector in the forward path. The pitch based formant estimation has four orders lower complexity and the simulation results achieve similar perceptual evaluation speech quality and added stable gain. Still the method provides slight degradation in the speech quality without voice activity detector. It also shows small and high frequency artifacts in the beginning of each sentence [18].

Some techniques project on the adaptive gain reduction algorithm method (i), in which the amount of gain reduction is variable, depending on the magnitude of feedback signal. This is only for low level signals and not for all input signals, which in turn, the gain of the desired signals are reduced. Also from method (ii), reducing the size of the vent may lead to occlusion effect, which may introduce another major problem.

The alternate method for adaptive feedback cancellation is the usage of notch filters. Whenever a whistling signal is detected, the algorithm generates sharp notch filter in order to suppress it. The notch filters are introduced at the feedback frequencies with the center frequency matching the peak frequency of feedback signal.

In a circuit, when only a few number of notch filters are generated by the algorithm to control the distortion in the acoustic environment, a frequency-hoping artifact is generated, as there is always an uncovered whistling feedback signal [12]. The notch filter method generally limits the gain reduction at the feedback frequencies.

Many companies offer several types of products from analog hearing devices (which has only fixed gain amplifier) to the digital based signal processing devices. Whenever there is a spectral overlap between the signal and noise or if the band occupied by the noise is unknown or varies with time, the use of conventional filters would lead to unacceptable distortion of the desired signal.

5. System Design. In many instances, the filtter characteristics needed to be variable, adapted to the signal charateristics or to be altered intelligently. In such cases, the coefficients of the filter must be varied and cannot be specified in advance. The feedback cancellation method preferred is through monitoring the transfer function of the feedback path. The transfer function of the signal is found, which is generated similar to the feedback path. Then the generated signal is subtracted from the output signal of the microphone circuit, so that the algorithm modifies the characteristics of the digital filter circuit. In such a way, the transfer function should have the similar frequency-amplitude-phase relationship corresponds to the feedback path.

A low- level noise signal is injected into the feedback path of the algorithm to estimate the transfer function of the corresponding signal, which is processed in multiple stages. Hence an adaptive filter is used in addition to a fixed filter. Initially, the noise signal that is sent to the receiver circuit of the hearing aid, which is then cross-correlated with the source output. This in turn estimates the transfer function of the feedback path. Thus the modelling of the feedback signal generates the cancellation signal. Generally a howl detector is used to monitor the changes in the feedback path above a particular frequency, refer Figure 5.1. A combination of adaptive and fixed filters are used to reduce the dynamic component of the feedback signal. A linear adaptive filter is chosen to control the variable parameters of the transfer function and those parameters can be adjusted by a suitable optimization algorithm.

The two major types of linear adaptive filters are (i) Least Mean Square(LMS) filter - reduces Mean Square Error while the input signals are stochastic. Under stationary environment, the error characteristics of the LMS filter has constant shape and orientation amd makes the filter charateistics to converge at or near the optimum point. The signal statistics (mean, variance and autocorrelation) are changed, once the weight values are converged, leads to readjustment of filter characteristics to the new optimum value.

Under non-stationary environment, the orientation and curvature of the error characteristics keep changing and make the filter characteristics to be converged slowly. This shows that the algorithm performs only for the seeking of the minimum point of the surface but tracking of changing position is also important to improve the performance of filter. This clears out that the LMS filtering is not suitable for real time or on-line filtering [13]. 


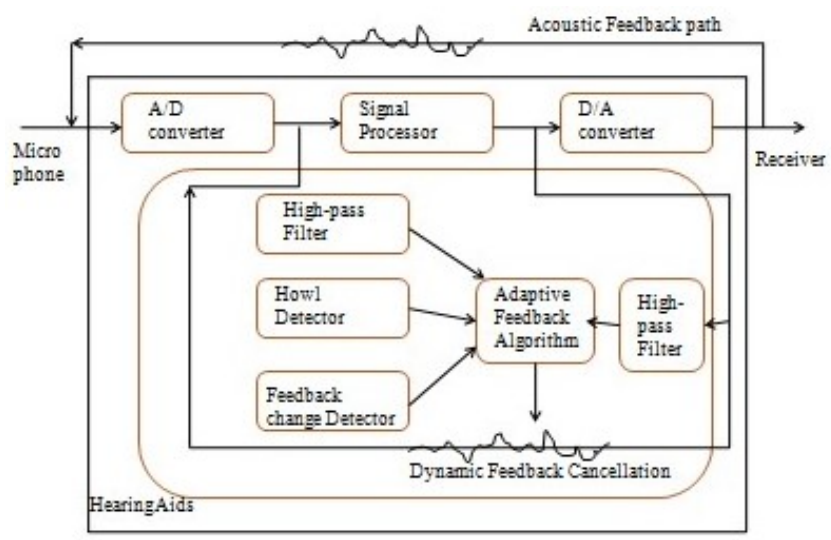

FiG. 5.1. Adaptive Feedback Algorithm

The Recursive Least Squares(RLS) filter minimizes the Weighted Linear Least Squares Cost Function. Also the input signals are deterministic and extremely Fast Convergence. The RLS filter is sensitive to computer round-off errors and eventually leads to instability.

5.1. Power Spectrum Estimation. The input noise voltages and the feedback signals generated in the hearing aid devices, can be modeled as random processes as most of the natural phenomena are characterized in terms of statistical averages. Random processes can be characterized by the estimation of spectral characteristics of signals [14].

Since the fluctuations in the signals are very random, the statistic characteristics of those signals are adopted. To characterize the random signals in time domain, the auto-correlation function is determined to yield the appropriate statistical average. The power density spectrum is obtained from the fourier transform of autocorrelation function, in order to transform the signals from the time domain to the frequency domain.

The estimation of power density spectrum of a signal from the observation of signal over a finite time interval is the basic problem to be considered as the short duration estimation limits the quality of power spectrum estimate. For statistically stationary signals, estimate is better for longer data record.

But when the signal statistics are non-stationary, it is difficult to select an arbitrarily long data record for the spetrum estimation. Therefore selecting a data record, as short as possible, allows to resolve the spectral characteristics of different signal components that have closely spaced frequency components.

To limit the duration of impulse response, windows are used in the FIR filter design. To say, to compute the spectrum of the signal, for the finite-duration sequence of $\mathrm{x}(\mathrm{n})$ is $0 \leq n \leq N-1$.

Convolution function smooths the spectrum $X(f)$, only when the window spectrum $W(f)$ is relatively narrower than $X(f)$. Rectangular windows smooth time-domain cut-off in addition they have side lobe leakage. Blackman window reduces lobe leakage but spectral width increased to $50 \%$ as it should be closely spaced spectra for a better estimate. That is, smooth time-domain windows reduces leakage, which in turn decreases the frequency resolution.

The finite-energy signals possess a fourier transform and are characterised in spectral domain by their energy density spectrum. Since the stationary random processes possess finite average power and they are characterised by the power density spectrum.

The leakage and frequency resolution problems provide the motivation for the power spectrum estimate methods based on non-parametric methods. This makes no assumptionof data generation and hence the estimate of power spectrum is obtained either through averaging or smoothing operations, which is performed directly on the periodogram or on the autocorrelation.

Bartlett method is a windowing technique based on averaging periodogram, used for reducing variance in the periodogram. The N-point sequence is sub-divided into L non-overlapping segments for each segment length is $M$. 


\begin{tabular}{|l|c|c|}
\hline & Bartlett PSE & Welch PSE \\
\hline Quality factor & $Q_{B}=1.0560$ & $\begin{array}{r}Q_{W}=0.7488, \text { for no overlap } \\
=1.3344, \text { for } 50 \% \text { overlap }\end{array}$ \\
\hline FFT length & $\mathrm{M}=15.0$ & $\mathrm{M}=21.3333$ \\
\hline No. Of FFTs & $\frac{N}{M}=1.0656$ & $\frac{2 N}{M}=1.4976$ \\
\hline No. of computation & $\frac{N}{2} \log _{2} \frac{0.9}{\Delta f}=468.864$ & $N \log _{2} \frac{1.28}{\Delta f}=666.8278$ \\
\hline
\end{tabular}

The periodogram is computed as:

$$
P_{x x}^{(i)}(f)=\frac{1}{M}\left|\sum_{n=0}^{M-1} x_{i}(n) e^{-j 2 \pi f_{n}}\right|^{2} \quad i=0,1, \cdots, L-1
$$

Hence the Bartlett power sectrum estimate is obtained by averaging the periodograms for the $L$ segments,

$$
P_{x x}^{B}(f)=\frac{1}{L}\left|\sum_{n=0}^{K-1} P_{x x}^{i}(f)\right|
$$

A window is obtained by reducing the length of data from $N$ points to $M=N / L$, and the spectral width is increased by a factor of $L$ and frequency resolution is decreased by factor $L$, in turn variance is reduced. Bartlett power spectrum estimate is asymptotically unbiased.

Welch method is based on averaging modified periodogram, originally derived from Bartlett window method. Two modifications made in Bartlett technique are: (i) Welch method allows the data segments to overlap, which is not done in Bartlett method. (ii) It windows the data segments prior to compute the periodogram, which results in Modified periodogram.

$$
P_{x x}^{(i)}(f)=\frac{1}{M U}\left|\sum_{n=0}^{M-1} x_{i}(n) w(n) e^{-j 2 \pi f_{n}}\right|^{2} \quad i=0,1, \cdots, L-1
$$

Hence in Bartlett method, the power sectrum estimate is computed by averaging the periodograms for the $L$ segments,

$$
P_{x x}^{W}(f)=\frac{1}{L}\left|P_{x x}^{(i)}(f)\right|
$$

Overlapping of the data segments can be varied for more or less than $50 \%$, in order to improve the relevant characteristics of the estimate [14]. On comparing the quality of Bartlett and Welch power spectrum estimates are given in Table 5.1.

The results obtained from Welch method is comparatively better than the Bartlett estimate. However the differences in performance are relatively small. The quality factor depends on the product of data length $\mathrm{N}$ and the frequency resolution f. From these results, it is concluded that Welch method requires a little more computational power than the Bartlett method. However the differences in performance are relatively small [14].

Characterization of the ambient noise has been carried out using the Power Spectrum Welch method (Figure 5.2). Simulation was carried out by sending the input signal with the noise data collected from the acoustic environment and used for denoising purpose.

The power spectrum estimation methods provide no idea about the data generation, when it is nonparametric. Bartlett windowing technique is one of the method, used to derive the power spectral estimation of a given signal. It provides smooth magnitude response in both passband and stopband, compared to other windowing techniques. Under which, the Welch method uses averaging modified periodogram technique. 


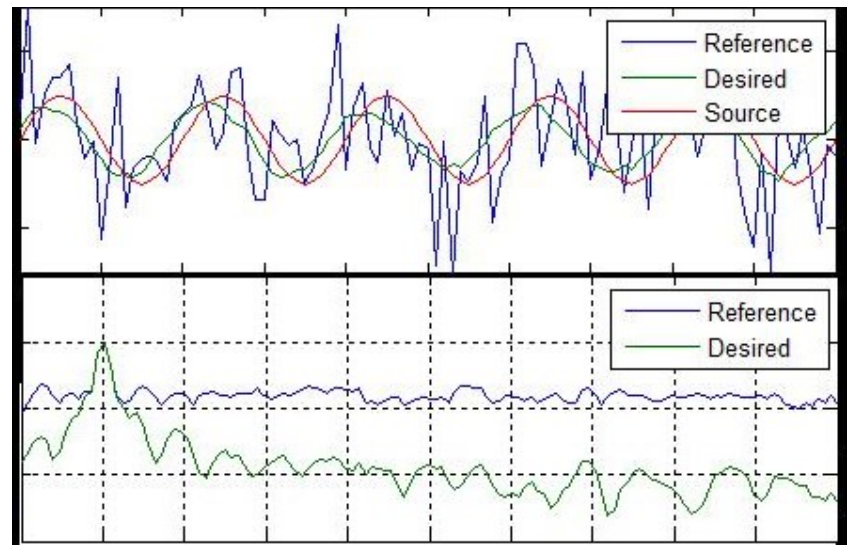

Fig. 5.2. Power Spectrum density of Noise Signal

6. Data Processing. The DSP based modern hearing aids have numerous parameters and fitting it, is a challenge for the audiologist after a comprehensive analysis. In order to select the algorithm that uses fewer resources, is implemented on the hearing device, based on low cost DSP. The vital feature of the assistive device, is to improve the signal to noise (SNR) under any type of background noises. The another important factor is to determine the memory requirements for the noise reduction methods to be used [6]. The DSP based hearing aids, functions for voice compression, noise reduction and dynamic equalization tasks to produce better results in hearing operations.

The digital processors process the incoming sounds received by a person with a particular hearing aid. The main features of a HA of a family are: (i) soft sound amplifier, which allows the user to perceive soft sounds related to weak or distant speech; (ii) Audibility extender is a vital function, which enables the user to hear sound, which is spectrally placed into the band where he has a problem. Also it is necessary to improve the convinience of the user. Hence the SNR ratio of the output signal is maximized, so that the sounds perceived by the person can be heard more naturally [6]. The hearing aids undergo classification of sounds signals and adaptation of signals among the noisy or quiet environments.

The filter coefficients of the FIR filter are obtained by Genetic Algorithm (GA) using the optimization procedure. The error signal is obtained by comparing the desired and the actual magnitude response, which is then minimized by GA. Also it is used to customize the specific subband in a particular octave in the hearing range of the hearing aid user.

7. Generic Operators. Genetic Algorithm (GA) is a class of probabilistic optimization algorithm and uses the concepts of 'Natural selection' and 'Genetic Inheritance', which is inspired by the biological evolution process. GA is simple, robust and generates Membership Functions. Hence it is more suitable for the complicated problems with little or unknown solution [4]. The flowchart of Genetic algorithm is shown in Figure 7.1.

The acoustic feedback available in the hearing circuit, is controlled or cancelled by varying the parameters, for fitting the system using genetic algorithm. To integrate the system of GA with perceptual feedback, an efficient search can be performed in a perceptual space is described by Eric A.Duant, etal [5]. This focuses on the selected features to be programmed on the DSP, to limit the computational facilities effectively. The algorithm parameters are used to control the processed incoming sound by the multitude of algorithm, which is then presented to the user.

The synthesizing of digital IIR filters using the real coded genetic algorithm (RCGA), is based on the real number representation of the chromosomes. The usage of real numbers allow us to extend the search domain by reducing the precision which has also the capacity to fine tune the solutions. Therefore comparing to binary coded strings, real numbers have more benefits in RCGA [7].

The RCGA is applied to solve the synthesis of digital filter whose magnitude response matches the human audiogram. The IIR filter exhibits significantly better performances than the FIR filter in approximating the 


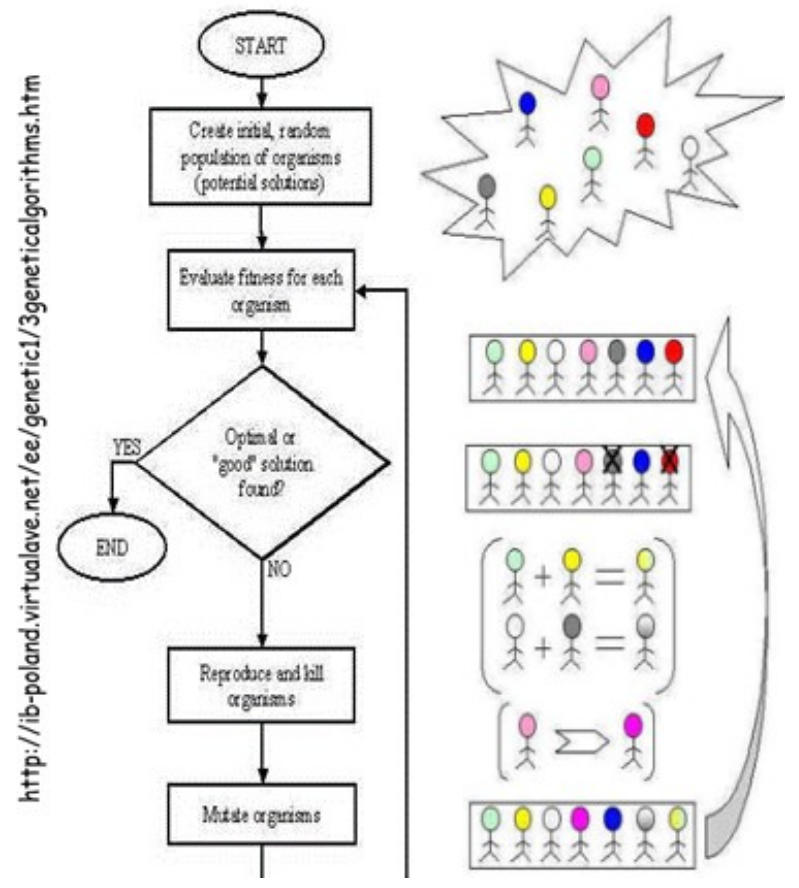

FIG. 7.1. Flowchart of Genetic Algorithm

required magnitude characteristic and in minimizing the resources necessary for the implementation [7]. The genetic algorithm is used with restricted search, is described by Enrique Alexandre [1], is explored for the above mentioned feature selection. The restricted search driven by the implemented GA performed better than both sequential methods and unconstrained genetic algorithms.

In general, optimatimization problems can be classified under (i) Combinatorial optimization problems in which Ant Colony optimization technique is used and (ii) Continous optimization problems in which Particle Swarm Intelligence is followed. The firefly algorithm is an another nature-inspired algorithm in GA, which is based on the concept of selecting the mating pairs under the point of attractiveness.

The GAs include the natural selection, mutation and other biological concepts, which can be applied to non-biological optimum based search problem. It generally starts from an initial population and using the evolutionary mechanism, the new members are produced by selection, crossover and mutation, shown in Figure 7.1.

The selection process is best performed by using Roulette wheel(RW) method, which is the most preferred fitness technique. The chromosomes that occupy larger area of the wheel and has more chances to be selected. It provides the area which is proportional to the fittness value. In RW selection, if the best chromosomes has the fitness of about $90 \%$, compared to other chromosomes, then all other will get very few chances to be selected. After performing selection, the new population can be obtained.

One or two genes are changed randomly by the mutation operator randomly with a predetermined probability. It yields insignificant results, when the mutation probability is low and it conducts the divergence of algorithm, when it is too high. Thus fixing the probability of mutation is considered as crucial. Concluding the experiments only with crossover operator than the mutation is ofcourse more effective.

The three different types of cross-over operators is shown through:

(i) single point crossover: the binary string value is copied from the beginning of the chromosome to the crossover point of one parent and the remaining is copied from the second parent. (Figure 7.2.a);

(ii) linear or uniform crossover: the binary bits are randomly copied from first or the second parent (Figure 7.2.b)

(iii) intermediate crossover: the binary string is copied from the beginning of chromosome of one parent to the 


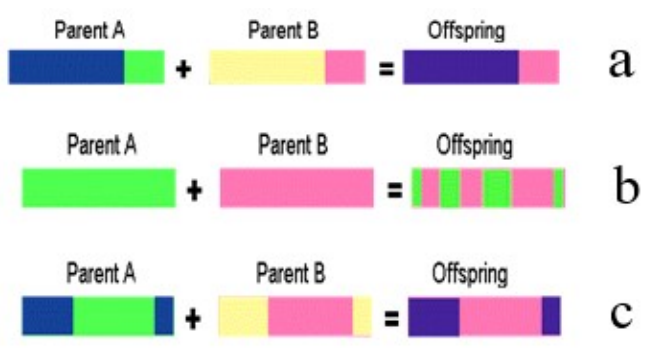

FIG. 7.2. Types of Crossover (a) Single Point Crossover (b) Linear Crossover (c) Intermediate Crossover

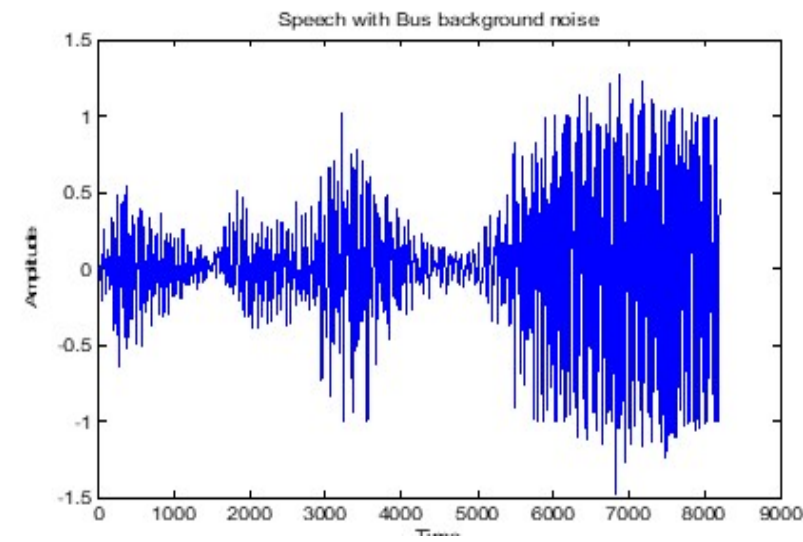

(a)

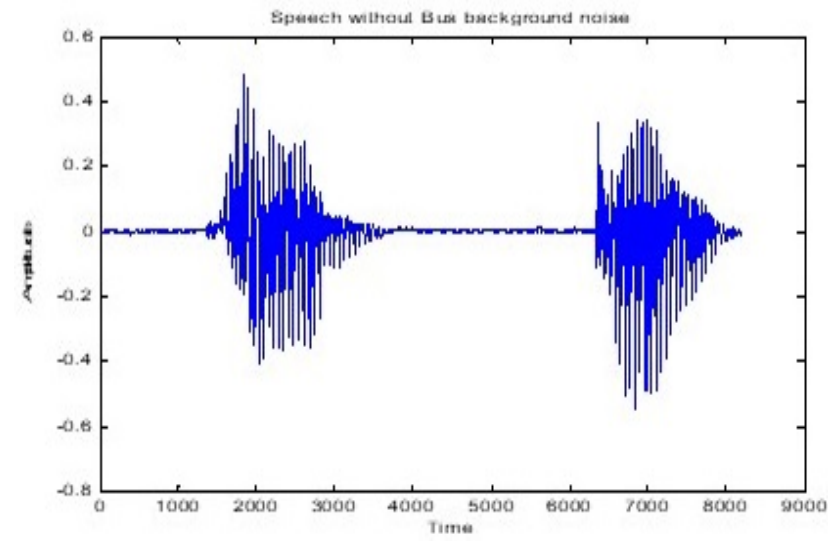

(b)

Fig. 8.1. (a) Speech with Bus Background Noise (b) Speech without Bus Background Noise

first crossover point, and the another part from the first to the second crossover point is copied from the second parent while the remaining string is copied from the parent on (Figure 7.2.c).

Therefore, in one generation, the total population fitness is changed and the same process is carried out again and again, until a stopping criterion is met. GA is based on Darwin's theory: the best ones should survive and propagate, based on their fitness. GA is used to learn or tune all the parameters of a network and its chromosomes encodes all the parameters of the model.

8. Simulation Results. The files correspond to speech in noise and speech in silence are considered under diverse environments. The data base contains certain number of files for training, validation and testing respectively. The speech signals are recorded from sources, with variable reverberational grades. Noise signals from stationary and non-stationary environments are collected and fed as a noise source. After following the perceptual criteria, signals with low SNRs are considered as noise and higher SNRs as clean speech signals [15].

To get more precise results, the input signal is divided into number of frames,with no overlap and with $50 \%$ overlap between the adjacent frames. The the modified periodogram using welch method is computed. Hence the mean and the variance are calculated in order to mitigate the values. The speech signal mixed with noise signal (here bus horn sound is considered as noise) is shown in Figure 8.1(a) and the noise is removed from which the clean speech is obtained, is shown in Figure 8.1(b). Matlab software is used to test the speech in noise and speech in silence.

The GA solver algorithm iterates the above steps, until a maximum number of generations are reached or till the lowest error value remains unchanged for a given number of iterations. The values obtained are found to be sufficient for the algorithm to converge properly.

The mixed signal (speech and the background noise signals) is given to the processor as input wave files. The algorithm which is used to reduce the noise level is stored in the specific processor (TMS320C6745) using Code 


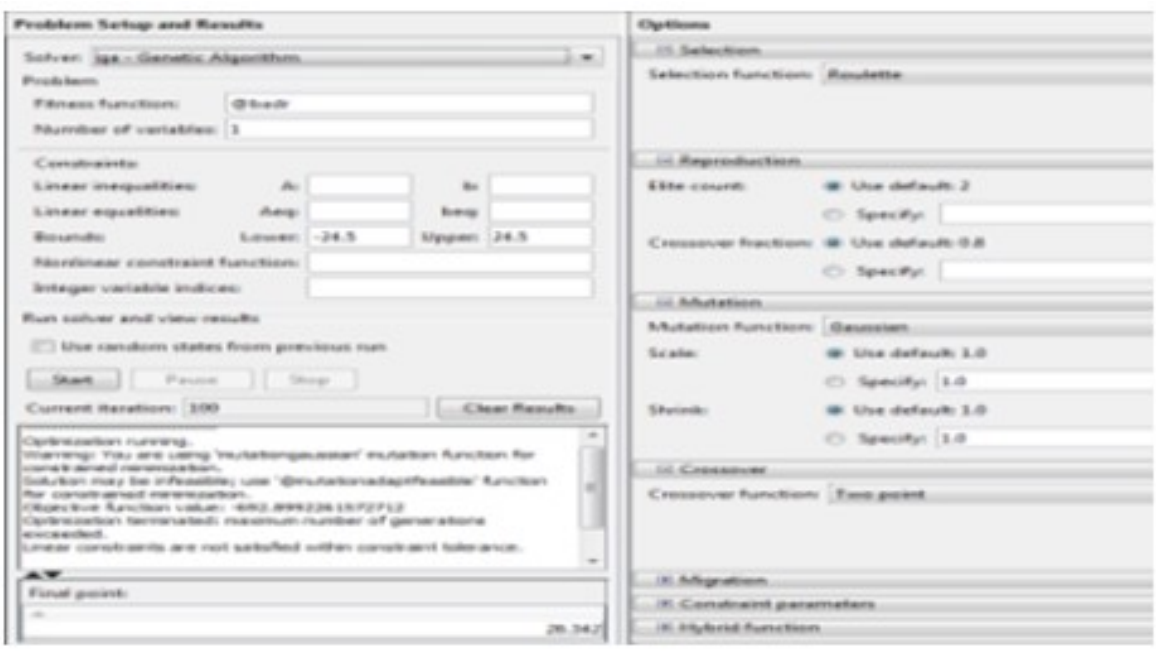

FiG. 8.2. GA Solver in Optimization Tool

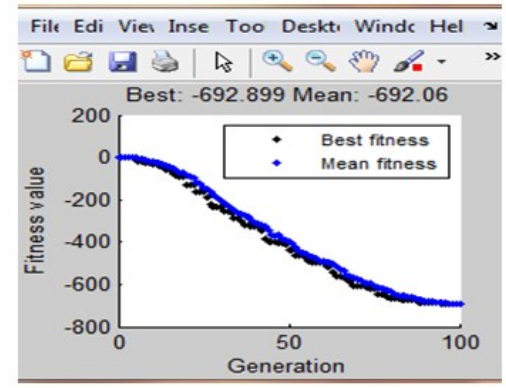

FIG. 8.3. Fitness Graph

Composer Studio (CCS). Based on this noise level, the volume of the hearing aid will be automatically adjusted by using GA solver in optimization toolbox. The processor will provide the desired result (speech signal) based on the input given to it. The fitness function can be created and minimized using genetic algorithm through the global optimization toolbox. The GA solver assumes one input $\mathrm{x}$, where $\mathrm{x}$ is a row vector elements as that of number of variables in the problem.

The value of the function is computed by the fitness function and it returns that scalar value in its one return argument b. It is minimized using GA by passing in a function that handles fitness function and also by mentioning the number of variables present in that problem. When there is average change in fitness value less than the options, then the optimization is terminated. The $\mathrm{x}$ value returned by the solver is the best point in the final population in genetic algorithm as shown in Figure 8.2.

To vectorize the fitness function, the GA solver is passed at one point of it. This is to speed up the process, by taking set of points and returning set of function values. Therefore in the fitness function, the vectored version of matrix $x$ with an arbitrary number of points as that of the rows of $x$ and it returns the column vector $y$ with the same number of rows $x$. When the average change in fitness value is less than the options provided, then the optimization is terminated and the fitness graph is shown in Figure 8.3.

9. Conclusion. The main intent of the paper is to address the issues related to Acoustic feedback effect in digital hearing aids and the possible methods available for cancellation. On the suitable database implementation, to evaluate the proposed algorithm, optimization tool is implemented to test the speech in noise and speech in silence in the acoustic environment. This paper presents an efficient sound signal separating from noise signal and the algorithm with very low computational cost for the speech in background noise for the 
hearing aid user. The genetic algorithm is useful in optimizing the bit distribution and executes by variation in the bit rate[9]. In order to improve the effectiveness of the proposed algorithm, the performance can also be validated by uniform distribution of bits technique. Improving the speech intelligibility, increases the comfort of the user and allows him to lead a normal life.

The design and the results obtained from the matlab simulation show the accuracy of hearing loss compensation and find the optimal coefficients. The proposed algorithm yields high levels of performance for different noises and SNR conditions but still it is not much sensitive to changes in the noise level. Tuning the algorithm with speech recorded in more diverse noise environments. Optimizing the DSP compatible ANSI-C code of the algorithm to ensure faster execution time. A certain number of listeners are required to justify the recordings containing the processing results to be tested, based on the features like Quality, Comprehension difficulty and Audibility of clipping.

\section{REFERENCES}

[1] Enrique Alexandre, Manuael Rosa and Fransico Lopez-Ferraras, Feature Selection for Soud Classification in Hearing Aids Through Restricted Search Driven by Genetic Algorithms, IEEE transactions on audio, speech and language, vol.15, No.8, pp.2249-2256, Nov 2007.

[2] S.Huang, L.Tian, Xiaojie Ma And Ying Wei, A Reconfigurable Sound Wave Decomposition Filterbank for Hearing Aids based on Nonlinear transformation, IEEE Transactions on biomedical circuits and systems, vol.10, No.2, pp.487-496, Apr 2016.

[3] Ying Wei, Yong Lian, A 16-Band Nonuniform FIR Digital Filterbank for Hearing Aid, IEEE, International Conference on Intelligent Data Communication Technologies and Internet of Things, pp. 186-189, 2006.

[4] Parmod Kumar, Alka Mahajan, Soft Computing Techniques for the Control of an Active Power Filter, IEEE Transactions on Power Delivery, vol.24, No.1, pp. 452-461, Jan 2009.

[5] Eric A.Durant, Gregory H.Wakefield, Dianne J.Van Tasell and Martin E.Rickert, Efficient Perceptual Tuning of Hearing Aids with Genetic Algorithms, IEEE Transactions on Speech and Audio Processing, vol.12, No.2, pp.144-155, March 2004.

[6] A.J.Uriz, P.D.Aguero, J.C.Moreira, J.C.Tulli And E.L.Gonzalez, Implementation of a noise reduction algorithm in a hearing aid device based on a dsPIC, IEEE Latin America Transactions, vol.11, No.1, pp.224-229, 2013.

[7] E.Szopos, I.SARacut, C. FArcas, M.NeAG, M.Topa, IIR Filter Synthesis Based on Real-Coded Genetic Algoritm for Human Audiogram Emulation, IEEE, 2015 International Symposium on Signals, Circuits and Systems (ISSCS), 2015.

[8] Jim Curran, A Forgotten Technique For Resolving The Occlusion Effect, Starkey Audiology Series, Vol 2, Issue 2 , 2012.

[9] David Ayllon, Roberto GilPita And Manuel Rosa-Zurera, Rate Constrained Source Separation for Speech Enhancement in Wireless- Communicated Binaural Hearing Aids, EURASIP Journal On Advances in Signal Processing, 2013.

[10] Amarita R And Bunyarit U, A New Selection Operator to Improve the Performance of Genetic Algorithm for Optimization Problems, Proceedings of IEEE, pp371-375, 2013.

[11] X.S.YANG AND S.DEB, Eagle Strategy using Levy walk and firefly algorithms for stochastic optimization, in Nature Inspired Coperative Strategies for Optimization, 2010, pp.101-111.

[12] King Chung, Trends in Amplification, in Westminster publications, vol.8, No.4, 2004.

[13] Maurice Bellanger, Adaptive Digital Filters, CRC Press, 2001.

[14] S. Salivahanan, A. Vallavaraj, Digital Signal Processing, Tata McGraw-Hill Education, 2001

[15] Lucas Cuadra, Enrique Alexandre, Lorena Alvarez and Manuael Rosa, Reducing the computational cost for Soud Classification in Hearing Aids by selecting features via Genetic Algorithms with Restricted Search, IEEE, 2008 International Conference on Audio, Language and Image Processing, pp.1320-1327, 2008.

[16] Soon-Suck Jarng, Carl Swanson, Frank Lee, Joseph Zou , Noise Reduction Algorithm applied for Hearing Aids, Proceedings in 3rd International Conference on Circuits, Control, Communication, Electricity, Electronics, Energy, System, Signal and Simulation (CES-CUBE), ASTL Vol. 25, pp. 261 - 266, 2013.

[17] A.Homayoun Kamkar-Parsi and Martin Bouchard, Improved Noise Power Spectrum Density Estimation for Binaural Hearing Aids Operating in a Diffuse Noise Field Environment, IEEE Transactions On Audio, Speech, And Language Processing, Vol. 17, No. 4, pp521 - 533, March 2009.

[18] Yi FanChiang, Cheng-Wen Wei, Yi-Le Meng, Yu-Wen Lin, Shyh-Jye Jou, Tian-Sheuan Chang, Low Complexity Formant Estimation Adaptive Feedback Cancellation for Hearing Aids Using Pitch Based Processing, IEEE/ACM Transactions on Audio, Speech, and Language Processing, Vol. 22, No. 8, pp. 1248 -1259, 2014.

Edited by: Swaminathan JN

Received: Nov 16, 2019

Accepted: Dec 4, 2019 